\title{
Increased Expression of miR-202-3p in Patients with Relapsing- Remitting Multiple Sclerosis
}

\author{
Majid Amini Khorasgani ${ }^{1}$, Parisa Mohammady Nejad ${ }^{* 2}$, Mohammad Mehdi Moghani Bashi ${ }^{3}$ \\ 1. Department of Genetics, Faculty of Basic Sciences, Shahrekord Islamic Azad University, Shahrekord, Iran. \\ 2. Department of Genetics, Faculty of Basic Sciences, Shahrekord Islamic Azad University, Shahrekord, Iran. \\ 3. Department of Genetics, Faculty of Basic Sciences, Kazerun Islamic Azad University, Kazerun, Iran.
}

Article Type:

Original Article

\section{Article History:}

Received: 6 Apr. 2018

Revised: 9 May. 2018

Accepted: 23 Jul. 2018

\section{*Correspondence:}

Parisa Mohammady

Nejad

Assistant Professor,

Department of

Genetics, Faculty of

Basic Sciences,

Shahrekord Islamic

Azad University,

Shahrekord, Iran.

Email:

parisa_mohamadynejad

@yahoo.com

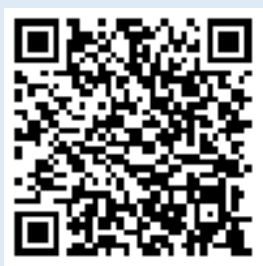

\begin{abstract}
Background and objectives: One of the latest studies in the genetics field is the evaluation of role of micro-RNAs as a biomarker for diagnosis of multiple sclerosis (MS), which is a demyelinating disease of the central nervous system (CNS) and emerges in the form of numerous small and large plaques in white matter in the brain and spinal cord. This disease could be associated with several complications, including reduced vision, spasticity and imbalance, and impaired sphincter control. MiR-202-3p is an intronic miRNA located in the ADA (Adenosine Deaminase) gene, which is the main enzyme involved in the pathway for the conversion of adenosine into inosine. Moreover, ADA regulates the inflammatory response and protection of tissue from damage as a strong complementary mechanism. This study aimed to evaluate the expression level of miR-202-3p in individuals diagnosed with relapsing-remitting multiple sclerosis (RRMS) and healthy individuals in Isfahan, Iran.
\end{abstract}

Methods: This analytical-observatory study was performed on 49 RRMS patients and 52 healthy individuals with no history of autoimmune and inflammatory diseases. Total RNA was extracted from blood lymphocytes by Ficoll and Trizol. Afterwards, cDNA was formed using a special miRNA cDNA kit, followed by the application of Real-time RT PCR to measure the expression of miR-202-3p in healthy individuals and patients.

Results: According to the results, the miR-202-3p expression was higher in patients, compared to healthy individuals $(\mathrm{P}=0.006)$. In addition, the sensitivity and diagnostic value of this miRNA in receiver operating characteristic (ROC) curve analysis were equal to $\mathrm{AUC}=0.80$ (area under the curve).

Conclusion: In line with other studies, our findings demonstrated that miR-2023p can be used as a biomarker in the diagnosis of MS. In addition, it seems that miR-202-3p acts as an immunosuppressant by inhibiting ADA gene, which regulates various processes related to inflammatory response and maintenance of tissue from damage.

Keywords: Multiple Sclerosis; RRMS; miR-202-3p; ADA Gene

\section{Introduction}

Multiple Sclerosis (MS) is a common progressive chronic and demyelinating disease of the central nervous system (CNS)
$(1,2)$, which leads to extensive destruction of the myelinated axons and formation of multiple plaques in the brain and spinal cord (3). The overall hypothesis about the development of this disease is that the

Copyright $\subseteq$ 2018, Jorjani Biomedicine Journal has published this work as an open access article under the terms of the Creative Commons Attribution License (http://creativecommons.org/licenses/by-nc/4.0/) which permits noncommercial uses of the work while it is properly cited. 
immune system is genetically damaged and cannot differentiate between the viral and myelin proteins in an individual's body, thereby producing antibodies that attack the body $(4,5)$. According to the global Atlas of $\mathrm{MS}$, the incidence of this disease is 30 per 100,000 (6). According to the reports, the prevalence of MS has been 5 per 35,100000 and 3 per 73/100000 during 2006-2011 (7).

The most common time for the emergence of the disease is during the second and third decade of a person's life between the ages of 25-30 years. In addition, the incidence of MS is higher in women than men with a ratio of 10:3 (8). While the cause of MS disease is unknown, some factors may lead to the development of MS, including DNA defects in nuclear and mitochondrial genomes, viral infections, hypoxia, oxidative stress, lack of light or vitamin D deficiency, and increased macrophages (microglia) and lymphocytes in the brain (9). MiR-202-3p, formerly known as hsa-miR-202-3p, is located within a chromosomal fragile site in 10q26 (10) and is a member of let-7 family (11). Moreover, MiR-202-3p is an intronic miRNA located in the ADA (Adenosine Deaminase) gene (Table 1).

While the expression of miR-202-3p decreases in breast cancer $(12,13)$, cervical squamous cell (14), colon cancer (15), follicular lymphoma (16), and gastric cancer $(17,18)$, it increases in multiple myeloma (19). ADA is the main enzyme involved in the pathway of adenosine breakdown into inosine (20-23). Generally, ADA is an important factor for the production of superoxide (through the control of the inosine concentration). In addition, it was observed that nucleoside phosphorylase turns inosine into hypoxanthine (a substrate for xanthine oxidase), which is involved in the production of superoxide during the conversion and transformation of purine substrates in macrophages (24). As a powerful and selective evolutionary mechanism, the adenosine system regulates different processes related to inflammatory response and protection of tissue from damage (25-28).

In humans, ADA activity is mainly detected in lymphoid tissues, including thymus, spleen, lymph nodes, and peripheral blood lymphocytes (29). This enzyme plays a role in the regulation and modification of performance of various types of immune cells (e.g., macrophages, lymphocytes, neutrophils, and dendritic cells) $(30,31)$. It is also observed that inflammatory cytokines (TNFa) are reduced and IL-10 anti-inflammatory cytokines are released by the activation of adenosine receptors (32-34). On the other hand, ADA deficiency is accompanied by a severe decrease in three main groups of lymphocytes, T cells, B cells, and NK cells. Evidence suggests that in this context, the metabolic basis of immune deficiency is probably related to the susceptibility of lymphocytes in adenosine accumulation (35).

There is no doubt about the necessity of the prevention or timely treatment of this complex disease. In addition, many of them have adverse complications, tolerating of which is extremely hard for patients. With the introduction of micro-RNAs as regulators of gene expression at the post-transcriptional level, another level of complexity in the regulation of genes expression has been revealed in the past two decades. Nonetheless, micro-RNAs have created high hopes for researchers in the field of genetics with their potential for treating complicated diseases, including cancer and autoimmune diseases, including MS. Research shows that while these small RNA molecules can be a disease- 
formation agent, they can be a treatment for diseases and provide a biomarker for evaluation of therapeutic efforts and disease progression (36).

One of the important steps in the development of the nervous system is the formation of synapses between the neural cells and the expression of specific proteins in the synapse region to increase neuronal communication. According to available data, some microRNAs are expressed in dendrites in particular and play an important role in neuronal development and synaptic plasticity $(37,38)$. Schwann cells in the peripheral nervous system and oligodendrocytes in the central nervous system play a major role in the formation of the myelin sheath, thereby significantly affecting the normal nerve function. The presence of myelin in myelinated axons causes mutated guidance and, as a result, prompt transmission of nerve signals. Current research has suggested the significant role of micro-RNAs in proliferation, differentiation and myelination by Schwann and Oligodendrocyte cells (39, 40).

Lack of Dicer1 enzyme, which is a key enzyme in the production of micro-RNAs, in mice is associated with severe impairment of myelin formation, which shows the role of micro-RNAs in the differentiation of Schwann and oligodendrocyte cells. Despite the fact that the Schwann cells and oligodendrocytes are responsible for myelination in the peripheral nerves and the central nervous system, respectively, some aspects of micro-RNAs expression and regulation are similar in these two types of cells.

This shows that similar micro-RNAs may play a role in differentiating myelinating cells (39, 41). Several micro-RNAs, including
miR-138, miR-219, miR-228 and miR-17992 micro-RNAs, are largely expressed in oligodendrocyte precursors and differentiate specific cells into oligodendrocytes by targeting them $(41,42)$. An abnormal formation or disturbance in maintaining myelin disturbs the direction of the neurological message and ultimately leads to nerve decay and impaired nerve functions (43). Therefore, since micro-RNAs play an important role in regulating the expression of genes at post-transcription levels in neurons and glial cells, changes in their expression in the nervous system lay the grounds for neurological diseases, such as neurodegenerative diseases (e.g., MS). With this background in mind, this study aimed to evaluate and compare the expression level of miR-202-3p in RRMS patients with healthy individuals collected from the MS clinic of Alzahra Hospital in Isfahan, Iran.

\section{Materials and Methods}

In this analytical and observational study, the confirmed and predicted target genes for hasmiR-202-3p were obtained using the (http://www.targetscan.org/vert_72/)

database. After that, 49 patients with RRMS, which was identified by lumbar puncture and MRI methods, along with 52 healthy individuals without a history of the disease, were selected from the MS clinic of Alzahra Hospital in Isfahan, which was confirmed by a neurologist. The questionnaire was completed after receiving informed consent from the participants (Table 2).

\section{RNA Extraction and cDNA Synthesis:}

A total of three $\mathrm{ml}$ of blood was collected from each subject in Ethylenediaminetetraacetic acid-containing tubes, followed by the extraction of PBMCs using French Biosera Ficol. Afterwards, RNA 
was extracted applying Sigma-Aldrich American Trizol, and the RNA quantity was assessed using the quantitative method of ultraviolet absorption spectrophotometric method by measuring the concentration and purity of the RNA sample extracted. The concentration of RNA extracted from samples was expressed in $\mathrm{ng} / \mu \mathrm{l}$, and the absorption ratio of 260/280 was measured using the Thermo 2000 CNano Drop Thermo American Thermo Device. In total, $2 \mu \mathrm{l}$ of this RNA was converted to cDNA using the Exiqon ${ }^{\circledR}$ miRCURY LNA TM Universal RT microRNA PCR kit of Exiqon Co., Denmark.

\section{Internal Control and Desired miRNA}

The housekeeping U6 gene, which is commonly used in miRNA expression studies, was selected as an internal control. After that, the stability of its expression and normalization of miR-202-3p expression was evaluated. It is notable that all sequence of primers was obtained from the miRanda site and was designed by Exiqon, Denmark.

\section{Real Time RT PCR}

RT PCR reaction was performed using the SYBR Green kit of the unique Asma assay in $10 \mu \mathrm{l}$ reactions. The final concentration of each of forward and reverse miR-202-3p primers and U6 gene was $0.3 \mu \mathrm{M}$. Fluorescence measurements were performed by $\mathrm{ABI}$ step one of ABI American company and data analysis was performed using SPSS software.

\section{Data Analysis}

Raw data from Real Time RT PCR was analyzed and reproductive efficiency and CT numbers were obtained for each reaction. CT (cycle threshold) refers to the number of cycles that a fluorescent signal must pass from the signal level of the field or threshold. The level of CT has a reverse association with the target nucleic acid concentration in the sample, meaning that the lower the CT level indicates the higher the target nucleic acid in the sample, and vice versa. In the next stage, the genetic variation of the study was analyzed using SPSS and T-test. In addition, P-value of less than 0.05 was considered significant, and the expression of the genes was recorded applying $2-\Delta \Delta \mathrm{CT}$ of the data as a multiple of the expression of miRNA, followed by its balancing by U6 internal control gene and interpretation in comparison with the normal sample.

\section{Results}

In this research, the mean of miR-202-3p expression was higher in the healthy group, compared to the patient group, which showed a significant difference in this regard $(\mathrm{P}=0.006) \quad$ (Diagram 1). Moreover, the sensitivity and diagnostic value of this miRNA in the (receiver operating characteristic) ROC curve analysis was equal to $\mathrm{AUC}=0.80$ (area under the curve) (Figure 2). 
Table1: The reaction between miR-202-3p and ADA gene in (http://www.targetscan.org/vert_72/)

\begin{tabular}{|c|c|c|c|c|c|}
\hline & & \multicolumn{3}{|c|}{$\begin{array}{l}\text { predicted consequential pairing of target } \\
\text { region (top) and miRNA (bottom) }\end{array}$} & $\begin{array}{l}\text { seed } \\
\text { match }\end{array}$ \\
\hline $\begin{array}{l}\text { Position } 154-160 \text { of } \mathrm{Al} \\
\text { hsa-miR-202 }\end{array}$ & A 3 UTR & \multicolumn{3}{|c|}{$\begin{array}{l}5^{*} \text {...UUUUCUGCACACACGUAUACCUCG... } \\
\text { IIIIIIII } \\
3^{*} \text { AAGGGUACGGGAUAUGGAGA }\end{array}$} & $\begin{array}{c}7 \mathrm{mer}- \\
\mathrm{m} 8\end{array}$ \\
\hline $\begin{array}{l}\text { site-type } \\
\text { contri-bution }\end{array}$ & \multicolumn{2}{|c|}{$\begin{array}{l}3 \text { pairing } \\
\text { contri-bution }\end{array}$} & $\begin{array}{l}\text { Iocal Au } \\
\text { contri-bution }\end{array}$ & \multicolumn{2}{|c|}{$\begin{array}{l}\text { Possifion } \\
\text { contri-bution }\end{array}$} \\
\hline-0.161 & \multicolumn{2}{|c|}{ o. 005} & 0.053 & \multicolumn{2}{|c|}{-0.023} \\
\hline $\begin{array}{l}\text { Comteret } \\
\text { secore }\end{array}$ & \multicolumn{2}{|c|}{$\begin{array}{l}\text { ontext score } \\
\text { percerntile }\end{array}$} & \multicolumn{2}{|c|}{$\begin{array}{l}\text { Conserved } \\
\text { ramch lemgith }\end{array}$} & $P C T$ \\
\hline-0.12 & \multicolumn{2}{|c|}{47} & \multicolumn{2}{|c|}{1.097} & N/A \\
\hline
\end{tabular}

Table 2: Clinical Information of Patients and Healthy Individuals

\begin{tabular}{lll}
\hline Characteristics & MS disease & Healthy individuals \\
\hline Number of samples & 49 & 52 \\
\hline Gender (N) & 28 women and 21 men & 28 women and 24 men \\
\hline Mean age (year) & $37.02 \pm 0.10$ & $33.86 \pm 10.57$ \\
\hline Mean age in days & & $30.64 \pm 8.40$ \\
\hline
\end{tabular}

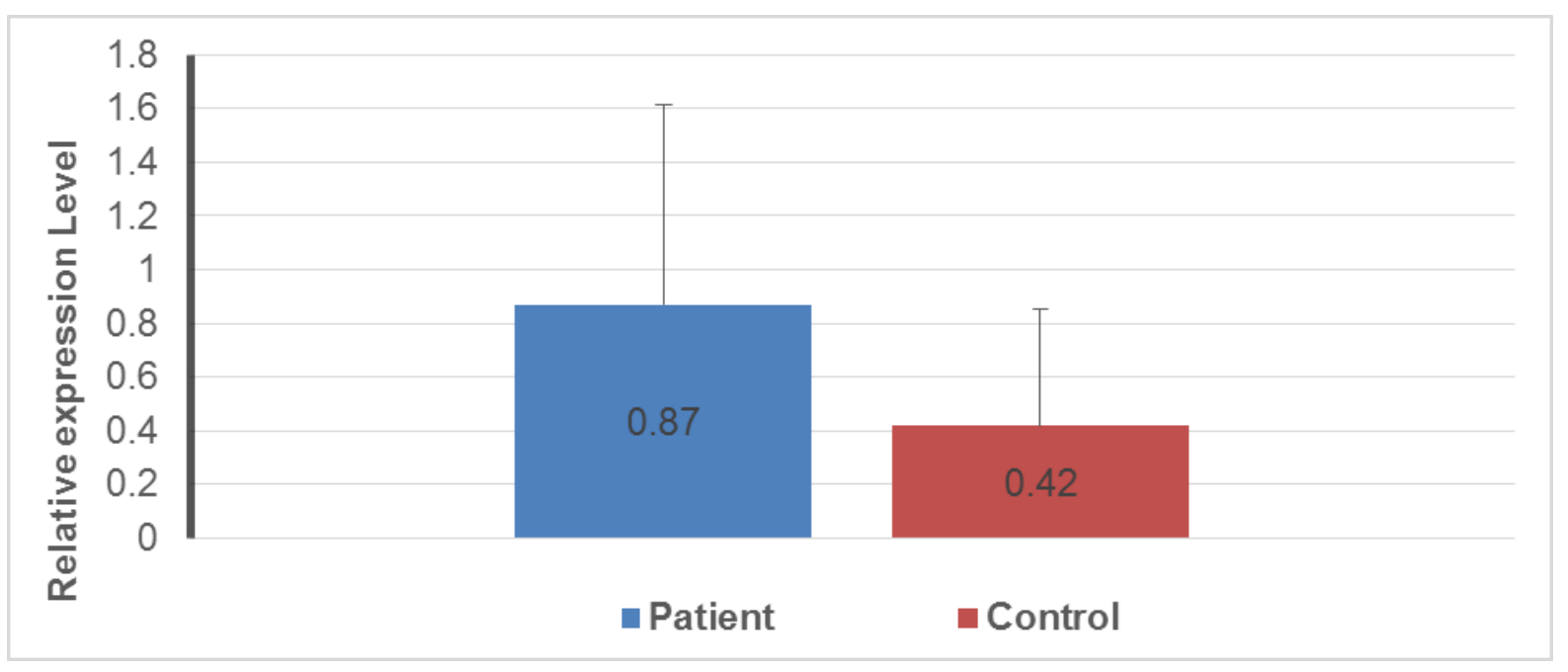

Diagram 1: Relative Expression Rate of miR-202-3p in healthy and patient groups 


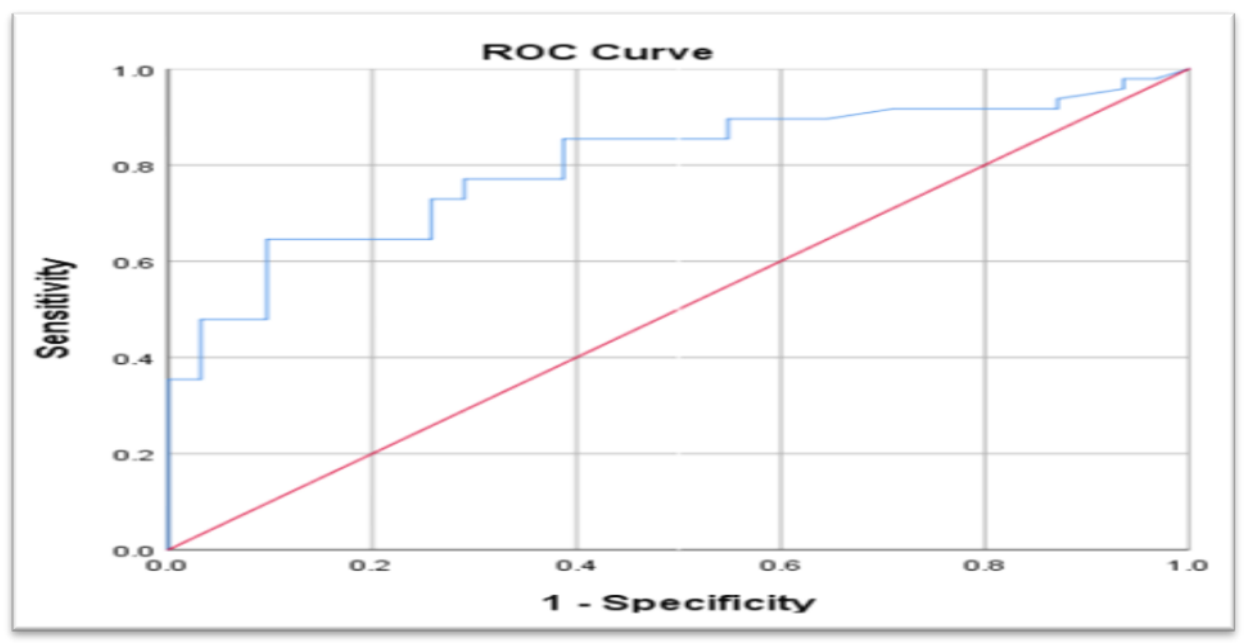

Diagram 2: ROC diagram, miR-202-3P in healthy and patient groups

\section{Discussion}

Multiple Sclerosis (MS) is a common progressive chronic and demyelinating disease of the CNS, and the majority of the young people, who constitute the workforce of the community, are affected by this disease. Unfortunately, in recent years, the rate of MS disease has been on the rise in Iran and, in particular, in Isfahan Province. The necessity of diagnosis, prevention, or timely treatments of this problem is not a secret. Common treatments for MS have not been able to function properly, and most of them have adverse effects that can be hardly tolerated by patients.

Over the past two decades, another level of complexity in the regulation of gene expression has been revealed with the arrival of micro-RNAs as regulators of gene expression at the post-transcription level. However, researchers have primarily focused on micro-RNAs owing to their potential abilities in the treatment of complicated diseases, including cancer and autoimmune diseases (e.g., MS). One of these miRNAs is MiR-202-3p located within a chromosomal fragile site in 10q26 (10) and miRNA is an intronic miRNA located in the ADA. ADA is a direct target for miR-202-3p, expression of which is reduced in breast cancer $(12,13)$, cervical squamous cell (14), colon cancer (15), follicular lymphoma (16), and gastric cancer $(17,18)$ and increased in multiple myeloma disease (19). According to the previous studies, ADA contributes to the regulation and modification of performance of numerous types of immune cells (e.g., macrophages, lymphocytes, neutrophils, and dendritic cells) $(30,31)$. As observed, the activation of adenosine receptors reduces the inflammatory cytokines (TNF-a) and promotes the release of IL-10 antiinflammatory cytokines (32-34). Therefore, this enzyme is an interesting drug target for managing multiple pathological conditions (e.g., inflammation, myocardial ischemic injury, and neurodegenerative disorders) (4446).

According to the results of the present study, there was a higher expression of miR-202-3p in patients, compared to the healthy subjects. Our findings are a report on the relationship 
between the expression of miR-202-3p and the risk of increased MS disease in Iran. It is notable that no similar study has been performed in other countries and other provinces of Iran that have similar nationality and ethnicity. The results of similar studies in the world have also yielded conflicting results. For instance, most micro-RNAs, such as miR-379-410 (encompassing more than 50 micro-RNA genes), are expressed in sets. Studies have shown that the miR-379-410 class is induced by neuronal depolarization and plays an important role in dendritic evolution in the cultured hippocampal neurons. According to some reports, miR134, which is one of the members of this group, is expressed in dendrites of cultured hippocampal neurons and in response to the brain-derived neurotrophic factor (BDNF), stimulating the growth of dendritic thorns by reversible inhibition of LiMK1 kinase mRNA (47).

In this condition, the miR-134 prevents the translation of mRNA encoding LiMK1 kinase, thereby playing a role in the synaptic evolution, maturation, and plasticity $(37,47)$. Moreover, NMDA-mediated neuronal stimulation increases the expression of miR219 , which is associated with alphaCaMKIImediated regulation of CA1 neuron excitability. It is hypothesized that miR-219 ultimately regulates the expression of NMDA receptors in the synapse, thereby regulating neural excitability $(42,43,48)$. Furthermore, Fischer et al. (1979) assessed the role of ADA in converting human monocytes into macrophages in the laboratory environment and reported a significant increase in ADA activity during the primary stages of the conversion of monocyte to macrophage. In addition, the highest increase in this enzyme was recorded after the first day in culture. This rapid increase in ADA activity denoted the role of this enzyme in the conversion process, indicating the existence of an effective mechanism in these cells for minimizing the effect of adenosine on the maturation phase.

In contrast, no change was observed in the Purine rescue path (such as adenine phosphoribosyltransferase or adenosine kinase) (49). In 1981, Tritsch and Niswander marked that there was a direct relationship between superoxide production intracellular ADA activity during phagocytosis in mouse macrophages. According to these results, it was suggested that ADA is an important factor in superoxide production (by controlling the concentration of inosine). In particular, it was observed that the nucleoside phosphorylase enzyme converts inosine into hypoxanthine (a substrate for xanthine oxidase), which contributes to the production of superoxide during the conversion and transformation of purinergic substrates in macrophages (24).

In line with this hypothesis, Yagawa and Okamura (1981) affirmed that ADA plays an active role in activating macrophage in guinea pigs. Especially, it was found that pharmacological blocking of ADA by coformycin led to decreased activation of macrophage. The possible relationship between ADA activity and chemotic/phagocytic stimulation of macrophages was evaluated in other studies (50). After exposing cells to an immunologic stimulus (opsonized zymosan), Tritsch and Iswaner (1985) reported a significant increase in ADA activity in Morin macrophage membranes and a higher enzymatic activity in the vicinity of phagocytic vacuoles, demonstrating the participation of $\mathrm{ADA}$ in macrophage response (51). 
In another study, Shaoqing JU et al. (2013) showed that the expression of miR-202-3p was significantly higher in the serum of multiple myeloma patients, compared to healthy subjects. It was concluded that miR202-3p acts as an immunosuppressant (19). In addition, Sun et al. (2014) demonstrated miR202-3p was used in patients with osteosarcoma by inhibiting the expression of Gli2 as a tumor suppressor to regulate cell proliferation and apoptosis (52). According to the results of the current research, the expression of miR-202-3p was higher in patients, compared to healthy individuals. It seems that this RNA inhibits ADA gene, which regulates various processes related to the inflammatory response and protection of tissue from damage, as a strong and selective complementary mechanism. In patients, the expression of this gene decreases and the

\section{Acknowledgements}

This article was extracted from a master's thesis. Hereby, we extend our gratitude to the patients and Dr. Etemadifar, who supplied the blood samples, for assisting us in performing the research.

\section{Declarations}

\section{References}

1. Rowland LP. Molecular genetics, pseudogenetics, and clinical neurology: The Robert Wartenberg Lecture. Neurology. 1983;33(9):1179-.

2. Olek M. Multiple sclerosis: etiology, diagnosis, and new treatment strategies: Springer; 2007.

3. Buc M. Role of regulatory $\mathrm{T}$ cells in pathogenesis and biological therapy of multiple sclerosis. Mediators of inflammation. 2013;2013. inflammatory cytokines (TNF-a) increases, which results in inhibition of release of IL-10 anti-inflammatory cytokines and conversion of adenosine to inosine. However, this gene is unable to reduce inflammation and tissue damages and disrupts the performance of $T$ and B cells. As such, miR-202-3p and ADA gene can be used to recognize and understand the pathology of MS disease.

It is suggested that more studies be conducted to more precisely assess the role of this miRNA in the occurrence of MS and its impact on other types of MS disease, such as PPMS, SPMS, and PRMS and determine the concomitant association between the expression and interaction between miR-202$3 p$ and ADA. It is noteworthy that the present paper was published in the Neurogenetics Congress of Iran.

\section{Conflict of interest}

We declare that we have no financial or non-financial conflicts of interest related to the subject matter or materials discussed in the article.

\section{Authors' contributions}

All authors contributed equally to this work.

4. Dutta R, Trapp BD. Pathogenesis of axonal and neuronal damage in multiple sclerosis. Neurology. 2007;68(22 suppl 3):S22-S31.

5. Minagar A, Alexander JS. Blood-brain barrier disruption in multiple sclerosis. Multiple Sclerosis Journal. 2003;9(6):540-9.

6. Alcalde-Cabero E, Almazán-Isla J, GarcíaMerino A, de Sá J, de Pedro-Cuesta J. Incidence of multiple sclerosis among European Economic Area populations, 1985-2009: the framework for monitoring. BMC neurology. 2013;13(1):58. 
7. Izadi S, Nikseresht A, Sharifian M, Sahraian MA, Jahromi AH, Aghighi M, et al. Significant increase in the prevalence of multiple sclerosis in iran in 2011. Iranian journal of medical sciences. 2014;39(2):152.

8. Ramagopalan SV, Dyment DA, Valdar W, Herrera BM, Criscuoli M, Yee IML, et al. Autoimmune disease in families with multiple sclerosis: a population-based study. The Lancet Neurology. 2007;6(7):604-10.

9. Van Horssen J, Witte ME, Schreibelt G, De Vries HE. Radical changes in multiple sclerosis pathogenesis. Biochimica et Biophysica Acta (BBA)-Molecular Basis of Disease. 2011;1812(2):141-50.

10. Farhana L, Dawson MI, Murshed F, Das JK, Rishi AK, Fontana JA. Upregulation of miR-150* and miR-630 induces apoptosis in pancreatic cancer cells by targeting IGF-1R. PloS one. 2013;8(5):e61015.

11. Huang Z, Huang S, Wang Q, Liang L, Ni S, Wang L, et al. MicroRNA-95 promotes cell proliferation and targets sorting Nexin 1 in human colorectal carcinoma. Cancer research. 2011.

12. Schreiber-Agus N, Chin L, Chen K, Torres R, Rao G, Guida P, et al. An amino-terminal domain of Mxi1 mediates anti-Myc oncogenic activity and interacts with a homolog of the yeast transcriptional repressor SIN3. Cell. 1995;80(5):777-86.

13. Gehring S, Rottmann S, Menkel AR, Mertsching J, Krippner-Heidenreich A, Lüscher B. Inhibition of proliferation and apoptosis by the transcriptional repressor Mad1 Repression of Fasinduced caspase- 8 activation. Journal of Biological chemistry. 2000;275(14):10413-20.

14. Hurlin P, Foley K, Ayer D, Eisenman R, Hanahan D, Arbeit J. Regulation of Myc and Mad during epidermal differentiation and HPVassociated tumorigenesis. Oncogene. 1995;11(12):2487-501.

15. Grandori C, Cowley SM, James LP, Eisenman RN. The Myc/Max/Mad network and the transcriptional control of cell behavior. Annual review of cell and developmental biology. 2000;16(1):653-99.
16. Lüscher B. MAD1 and its life as a MYC antagonist: an update. European journal of cell biology. 2012;91(6-7):506-14.

17. Jiang Z, Guo J, Xiao B, Miao Y, Huang R, Li $\mathrm{D}$, et al. Increased expression of miR-421 in human gastric carcinoma and its clinical association. Journal of gastroenterology. 2010;45(1):17-23.

18. Zhao Y, Li C, Wang M, Su L, Qu Y, Li J, et al. Decrease of miR-202-3p expression, a novel tumor suppressor, in gastric cancer. Plos one. 2013;8(7):e69756.

19. Yu J, Qiu X, Shen X, Shi W, Wu X, Gu G, et al. miR-202 expression concentration and its clinical significance in the serum of multiple myeloma patients. Annals of clinical biochemistry. 2014;51(5):543-9.

20. Ratech H, Thorbecke GJ, Meredith G, Hirschhorn R. Comparison and possible homology of isozymes of adenosine deaminase in Aves and humans. Enzyme. 1981;26:74-84.

21. Ungerer J, Oosthuizen H, Bissbort S, Vermaak $\mathrm{W}$. Serum adenosine deaminase: isoenzymes and diagnostic application. Clinical Chemistry. 1992;38(7):1322-6.

22. Maier SA, Galellis JR, McDermid HE. Phylogenetic analysis reveals a novel protein family closely related to adenosine deaminase. Journal of molecular evolution. 2005;61(6):77694.

23. Zavialov AV, Engström ̊. Human ADA2 belongs to a new family of growth factors with adenosine deaminase activity. Biochemical Journal. 2005;391(1):51-7.

24.Tritsch GL, Niswander PW. Adenosine deaminase activity and superoxide formation during phagocytosis and membrane perturbation of macrophages. Immunological communications. 1981;10(1):1-7.

25. Sitkovsky MV, Ohta A. The 'danger'sensors that STOP the immune response: the A2 adenosine receptors? Trends in immunology. 2005;26(6):299-304.

26. Haskó G, Cronstein BN. Adenosine: an endogenous regulator of innate immunity. Trends in immunology. 2004;25(1):33-9. 
27. Fredholm B. Adenosine, an endogenous distress signal, modulates tissue damage and repair. Cell death and differentiation. 2007;14(7):1315.

28. Antonioli L, Fornai M, Colucci R, Ghisu N, Tuccori M, Del Tacca M, et al. Regulation of enteric functions by adenosine: pathophysiological and pharmacological implications. Pharmacology \& therapeutics. 2008;120(3):233-53.

29. Daddona PE. Human adenosine deaminase. Properties and turnover in cultured $\mathrm{T}$ and $\mathrm{B}$ lymphoblasts. Journal of Biological Chemistry. 1981;256(23):12496-501.

30. Aldrich MB, Blackburn MR, Kellems RE. The importance of adenosine deaminase for lymphocyte development and function. Biochemical and biophysical research communications. 2000;272(2):311-5.

31. Franco R, Pacheco R, Gatell JM, Gallart T, Lluis C. Enzymatic and extraenzymatic role of adenosine deaminase 1 in T-cell-dendritic cell contacts and in alterations of the immune function. Critical Reviews ${ }^{\mathrm{TM}}$ in Immunology. 2007;27(6).

32. Hasko G, Szabó C, Németh ZH, Kvetan V, Pastores S, Vizi ES. Adenosine receptor agonists differentially regulate IL-10, TNF-alpha, and nitric oxide production in RAW 264.7 macrophages and in endotoxemic mice. The Journal of Immunology. 1996;157(10):4634-40.

33. Németh ZH, Lutz CS, Csóka B, Deitch EA, Leibovich SJ, Gause WC, et al. Adenosine augments IL-10 production by macrophages through an A2B receptor-mediated posttranscriptional mechanism. The Journal of Immunology. 2005;175(12):8260-70.

34. Csóka B, Németh ZH, Virág L, Gergely $P$, Leibovich SJ, Pacher $\mathrm{P}$, et al. A2A adenosine receptors and $\mathrm{C} / \mathrm{EBP} \beta$ are crucially required for IL-10 production by macrophages exposed to Escherichia coli. Blood. 2007;110(7):2685-95.

35. Blackburn MR, Kellems RE. Adenosine deaminase deficiency: metabolic basis of immune deficiency and pulmonary inflammation. Advances in immunology. 86: Elsevier; 2005. p. $1-41$.
36. Nathan C. Points of control in inflammation. Nature. 2002;420(6917):846.

37. Schratt GM, Tuebing F, Nigh EA, Kane CG, Sabatini ME, Kiebler M, et al. A brain-specific microRNA regulates dendritic spine development. nature. 2006;439(7074):283.

38. Cohen JE, Lee PR, Chen S, Li W, Fields RD. MicroRNA regulation of homeostatic synaptic plasticity. Proceedings of the National Academy of Sciences. 2011;108(28):11650-5.

39.Dugas JC, Notterpek L. MicroRNAs in oligodendrocyte and Schwann cell differentiation. Developmental neuroscience. 2011;33(1):14-20.

40. Barca-Mayo O, Lu QR. Fine-tuning oligodendrocyte development by microRNAs. Frontiers in neuroscience. 2012;6.

41. He $\mathrm{X}, \mathrm{Yu} \mathrm{Y}$, Awatramani $\mathrm{R}, \mathrm{Lu} \mathrm{QR}$. Unwrapping myelination by microRNAs. The Neuroscientist. 2012;18(1):45-55.

42. Zhao X, He X, Han X, Yu Y, Ye F, Chen Y, et al. MicroRNA-mediated control of oligodendrocyte differentiation. Neuron. 2010;65(5):612-26.

43. Svaren J. MicroRNA and transcriptional crosstalk in myelinating glia. Neurochemistry international. 2014;77:50-7.

44. Lasley RD, Mentzer RM. Myocardial protection: the adenosine story. Drug development research. 1996;39(3-4):314-8.

45. Cristalli G, Costanzi S, Lambertucci C, Lupidi $\mathrm{G}$, Vittori S, Volpini R, et al. Adenosine deaminase: functional implications and different classes of inhibitors. Medicinal research reviews. 2001;21(2):105-28.

46. Honma Y. A novel therapeutic strategy against monocytic leukemia with deoxyadenosine analogs and adenosine deaminase inhibitors. Leukemia \& lymphoma. 2001;42(5):953-62.

47. Muddashetty R, Bassell GJ. A boost in microRNAs shapes up the neuron. The EMBO journal. 2009;28(6):617-8.

48. Li J-S, Yao Z-X. MicroRNAs: novel regulators of oligodendrocyte differentiation and potential therapeutic targets in demyelination- 
related diseases. Molecular neurobiology. 2012;45(1):200-12.

49. Fischer D, Van der Weyden MB, Snyderman $\mathrm{R}$, Kelley WN. A role for adenosine deaminase in human monocyte maturation. The Journal of clinical investigation. 1976;58(2):399-407.

50. Yagawa K, Okamura J. Role of adenosine deaminase in activation of macrophages. Infect Immun. 1981;32(1):394-7.
51. Tritsch GL, Niswander PW. Purine catabolism as a source of superoxide in macrophages. Ann N Y Acad Sci. 1985;451:279-90.

52. Sun Z, Zhang T, Hong H, Liu Q, Zhang H. miR-202 suppresses proliferation and induces apoptosis of osteosarcoma cells by downregulating Gli2. Mol Cell Biochem. 2014;397(1-2):277-83.

\section{How to cite:}

Amini Khorasgani M, Mohammady Nejad P, Moghani Bashi MM. Increased Expression of miR-202-3p in Patients with Relapsing-Remitting Multiple Sclerosis. Jorjani Biomedicine Journal. 2018; 6(4): 8-18. 